\title{
Non-Linear Effects in Asymmetric Reactions: Negative and Positive Stereoselectivity in the Formation of Homoleptic 1:2 Metal to Ligand Complexes of Chiral 1,9-Disubstituted Semicorrin Ligands
}

\author{
Nathalie Guicher, Helen Stoeckli-Evans, and Klaus Bernauer*
}

\begin{abstract}
The stereoselectivity of the formation of bis-chelates with the 1,9-disubstituted chiral semicorrin ligands (1S,9S)-dimethyl 5-cyanosemicorrin-1,9-dicarboxylate (1) for $\mathrm{Co}^{2+}, \mathrm{Ni}^{2+}, \mathrm{Cu}^{2+}$, and $\mathrm{Pd}^{2+}$ and with its $(1 S, 9 S)$-diethyl analogue 2 for $\mathrm{Co}^{2+}$ and $\mathrm{Cu}^{2+}$ have been measured by the method of continuous variation of enantiomers, and with 1 for $\mathrm{Zn}^{2+}$ by NMR. Positive selectivities were found for $\mathrm{Co}^{2+}, \mathrm{Ni}^{2+}$, and $\mathrm{Zn}^{2+}$ complexes, whereas very high negative selectivities prevail in the complexes of $\mathrm{Cu}^{2+}$ and $\mathrm{Pd}^{2+}$. These results are rationalised by $\mathrm{X}$-ray structural determinations of some of the corresponding complexes. The optically active $\mathrm{Cu}^{2+}$ and $\mathrm{Pd}^{2+}$ complexes show distorted square planar structures with chirality predetermined by the chirality of the ligand. $\mathrm{Ni}^{2+}$ complexes are five or hexacoordinated and exhibit a distorted tetrahedral arrangement of the four coordinated nitrogen atoms. In the optically active compound the ester moiety is coordinated by the oxygen atom of the alkoxy group and the arrangement of the two N-N chelate rings shows $\Delta-(S, S)$ chirality. Interestingly, with the racemic ligand, it is not the more stable heterochiral, but the racemic complex containing the two homochiral enantiomers which is obtained. The coordination of the ester groups occurs by the carbonyl oxygen and the chirality is opposite to the optically active compound, e.g $\Lambda(S, S) / \Delta-(R, R)$. The structure of the optically active zinc compound shows very weak interaction with the alkoxy group of one ester moiety of each ligand molecule and the chirality of the $N$-N-chelate rings is $\Lambda$-(S,S). Again, the compound obtained with the racemic ligand contains the enantiomers of the homochiral complex, the chirality of which is $\Lambda-(R, R) / \Delta-(S, S)$. The results are discussed with respect to the possibility to perform asymmetric amplifications of the 1:1 complexes of semicorrin ligands as enantioselective catalysts.
\end{abstract}

Keywords: Asymmetric reactions · Negative and positive stereoselectivity $\cdot$ Non-linear effects

\section{Introduction}

In asymmetric reactions the enantiomeric excess (\%ee) usually depends linearly upon the enantiomeric purity of the stereoselective catalyst or reagent. In several cases however, non-linear effects, first described in 1986 by Kagan and coworkers have been

\footnotetext{
${ }^{*}$ Correspondence: Prof. K. Bernauer Institute de Chimie

Université de Neuchâte

Av. de Bellevaux 51

C.P. 2

CH-2007 Neuchâtel

Tel.: +413271824 85

Fax: +41327182511

E-Mail: klaus.bernauer@unine.ch
}

observed [1]. In order to explain these phenomena Kagan suggested mainly two models: the $\mathrm{ML}_{\mathrm{n}}$ and the reservoir model [2]. Both models are based on the non-statistical distribution between the heterochiral $[\mathrm{M}(R)(S)]$ and the homochiral, optically active $[\mathrm{M}(R, R)]$ and $[\mathrm{M}(S, S)]$ molecular forms according to Scheme 1 . In the former the non-linearity can for example occur due to different stability and/or reactivity of the homo and the heterochiral forms, whereas in the latter only the $1: 1$ species is catalytically active. A non-statistical distribution in the $1: 2$ complexes then yields a $[\mathrm{M}(R)] /$ $[\mathrm{M}(S)]$ ratio different from that of the free ligand. Used as catalyst or as a reagent, the enantiomeric excess in the product can be higher than expected from enantiomeric purity of the reacting system. This is known as asymmetric amplification. Until now, most studies focused on non-linear effects with the aim to unravel reaction mechanisms based on Kagan's model [3]. More recently, the kinetic behaviour, in addition to thermodynamic effects, has been taken into account [4]. Despite the interest in non-linear effects observed in enantioselective reactions, quantitative analyses of the relationship between optical purities of the products and the reacting species are almost inexistent. In a first study this relationship has been reported for the reduction of acetophenone by $\beta$-chlorodiisopinocamphenylborane [5]. 


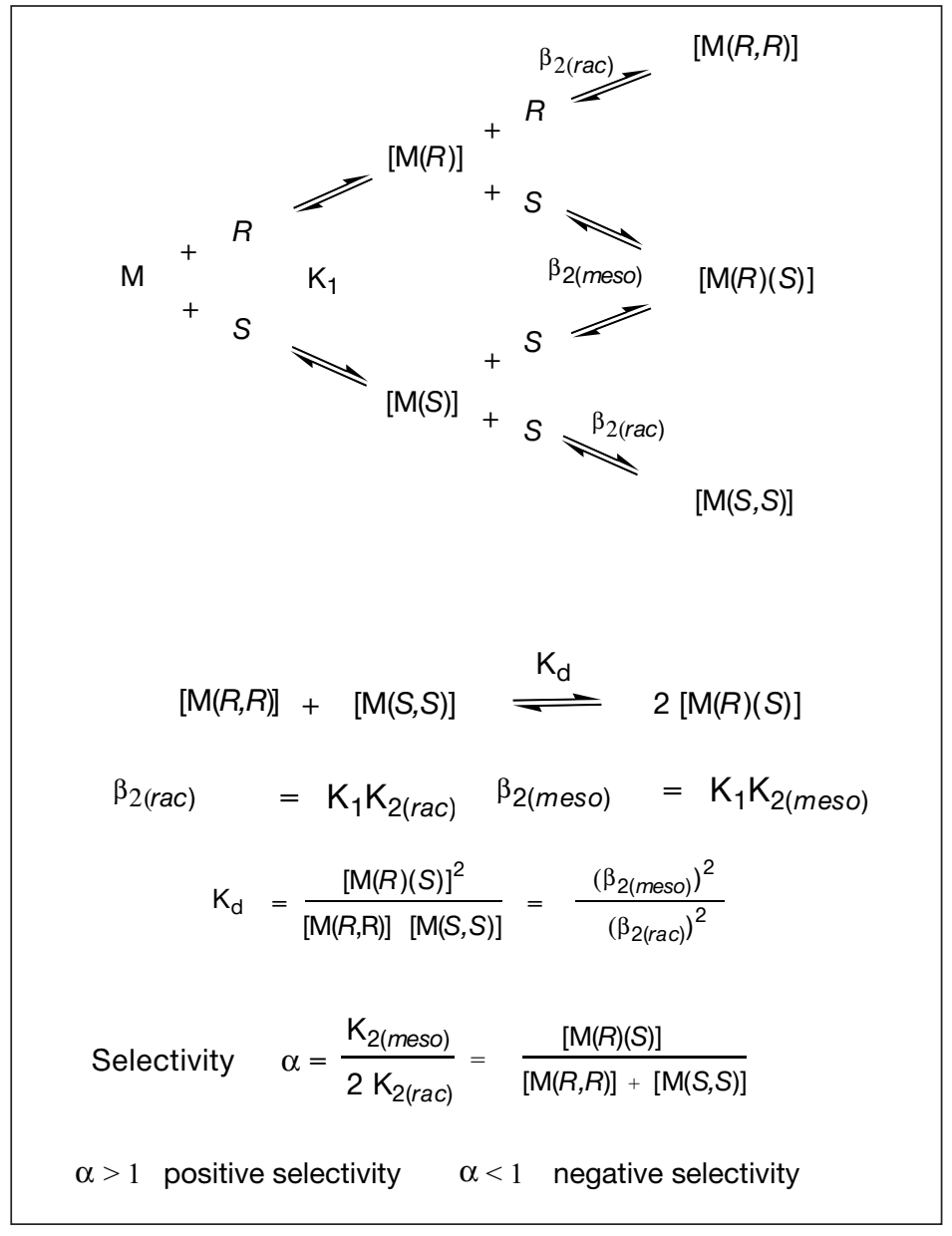

In that case the reagent is formed in an non-reversible way using different ratios of $(+)-$ and $(-)-\alpha-$ pinene. Similar studies involving labile metal complexes as the catalytic system are not known. In the present communication we report stereoselectivity measurements of the complex formation of $\mathrm{Co}^{2+}, \mathrm{Ni}^{2+}, \mathrm{Cu}^{2+}, \mathrm{Zn}^{2+}$, and $\mathrm{Pd}^{2+}$ with the chiral semicorrines $(1 S, 9 S)$-dimethyl 5cyanosemicorrin-1,9-dicarboxylate (1) and its $(1 S, 9 S)$-diethyl analogue 2, a ligand system developed by Pfaltz [6], frequently used in asymmetric catalysis. The selectivity data are compared to structural properties of some of the corresponding solid compounds.

\section{Stereoselectivity}

Stereoselectivity of the 1:2 metal to ligand complexes of 1,9-disubstituted semicorrin ligands $\mathbf{1}$ and $\mathbf{2}$ with the bivalent metal ions $\mathrm{Co}^{2+}, \mathrm{Ni}^{2+}, \mathrm{Cu}^{2+}$, and $\mathrm{Pd}^{2+}$ were determined by circular dichroism (CD) measurements using the method of continuous variation of enantiomers [7][8]. Corresponding values for $\mathrm{Zn}^{2+}$ were obtained from NMR spectra of 1:2 mixtures of anhydrous $\mathrm{Zn}\left(\mathrm{CH}_{3} \mathrm{COO}\right)_{2}$ with either optically active or the racemic 1 in $\mathrm{CD}_{3} \mathrm{OD}$. The results are given in the Table.

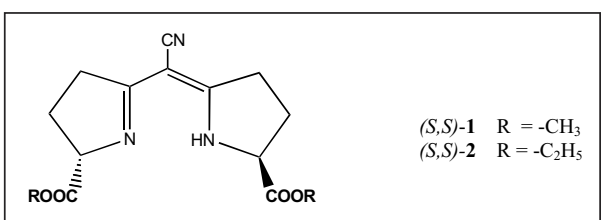

According to the definition in Scheme 1 , the $\mathrm{Co}^{2+}, \mathrm{Ni}^{2+}$, and $\mathrm{Zn}^{2+}$ complexes show moderately positive selectivity, $\mathrm{Cu}^{2+}$ and $\mathrm{Pd}^{2+}$ strongly negative selectivity. Whereas in the case of positive selectivity $(\alpha>1)$ the magnitude of the difference of the measured physicochemical parameter used for the determination (e.g. $\mathrm{pH}, \mathrm{CD}$, etc.) allows a precise determination of the $\mathrm{K}_{\text {meso }} /\left(2 \cdot \mathrm{K}_{\text {rac }}\right)$ ratio, this is not true in the case of negative selectivity $(\alpha<1)$, for which the differences are rather small and reach a limiting value for $\alpha \rightarrow 0$. This prob- lem in the determination of negative stereoselectivity of homoleptic bis-complexes is a consequence of the $\Delta \Delta \mathrm{G}$ value between the statistical distribution of homo- and heterochiral species and the pure homochirality, equal to $-\mathrm{RT} \cdot \ln 2$, e.g. $1.72 \mathrm{~kJ} \mathrm{~mol}^{-1}$ at $25{ }^{\circ} \mathrm{C}$. In the case of positive stereoselectivity such a limit does not exist (for a detailed discussion, see [7]).

The differences measured for the complexes of $\mathrm{Cu}^{2+}$ and $\mathrm{Pd}^{2+}$ with $\mathbf{1}$ are very close to the theoretical maximum, and the values given in the Table have to be considered as upper limits. The real stereoselectivity with these two metal ions might therefore be higher than indicated, which means that the heterochiral form of the bis-complex is present at less than $5 \%$ in the equilibrium mixture.

It seems reasonable to admit that the change from positive to negative stereoselectivity is a consequence of a different arrangement of the ligand molecules in the coordination sphere of the metal ion. In the case of tetra-coordination, two basic geometries are possible, square planar and tetrahedral. The schematic representation in Scheme 2 makes clear that for chiral $\mathrm{C}_{2}$ symmetric ligands the homochiral species is favoured for the square planar arrangement, whereas the heterochiral isomer should be more stable in the tetrahedral arrangement.

\section{X-ray Structural Determinations}

The data given in the Table suggest square planar structures for $\mathrm{Cu}^{2+}$ and $\mathrm{Pd}^{2+}$ complexes and tetrahedral structures for the $\mathrm{Co}^{2+}, \mathrm{Ni}^{2+}$, and $\mathrm{Zn}^{2+}$ complexes. In order to examine this hypothesis, we determined the X-ray molecular structure of some of the $\left[\mathrm{M}(\mathbf{1})_{2}\right]$ complexes obtained with both the optically active and the racemic ligand.

The crystal structure of the copper complex with the analogous ligand $(1 S, 9 S)-1,9-$ bis(1-hydroxy-1-methylethyl)semicorrin-5carbonitrile has been published [6]. The structure is described as tetrahedrally distorted with the two ligand planes tilted rel-

Table. $\alpha$-Values of $\mathrm{ML}_{2}$ complex formation of $\mathbf{1}$ and $\mathbf{2}$ with various metal ions $\left(\alpha=\mathrm{K}_{\text {meso }} /\left(2 \cdot \mathrm{K}_{\text {rac }}\right)\right)$

\begin{tabular}{lcccc}
$\mathrm{M}^{2+}$ & $\mathrm{L}=\mathbf{1}$ & $\mathrm{L}=\mathbf{2}$ & $\%[\mathrm{M}((R, R)((\mathrm{S}, S)-\mathbf{1})]$ & $\begin{array}{c}\%\left[\mathrm{M}((S, S)-\mathbf{1})_{2}\right]= \\
\%\left[\mathrm{M}((R, R)-1)_{2}\right]\end{array}$ \\
$\mathrm{Co}^{2+}$ & 2.1 & 8.1 & 67.4 & 16.2 \\
$\mathrm{Ni}^{2+}$ & 2.8 & - & 73.6 & 13.2 \\
$\mathrm{Cu}^{2+}$ & $<0.05$ & 0.17 & $<4.8$ & $>47.6$ \\
$\mathrm{Zn}^{2+}$ & 3.7 & & 78.8 & 10.6 \\
$\mathrm{Pd}^{2+}$ & $<0.06$ & - & $<5.6$ & $>47.2$ \\
\multicolumn{7}{l}{} \\
a from NMR measurements.
\end{tabular}




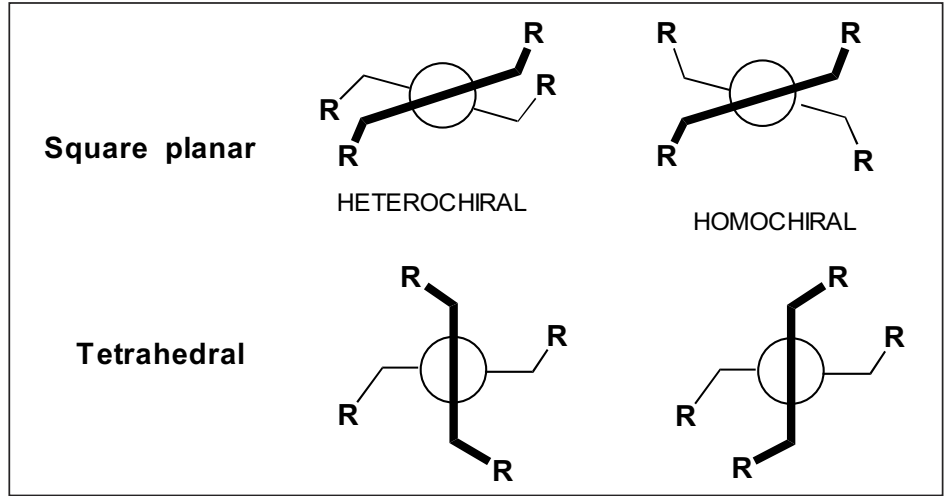

Scheme 2

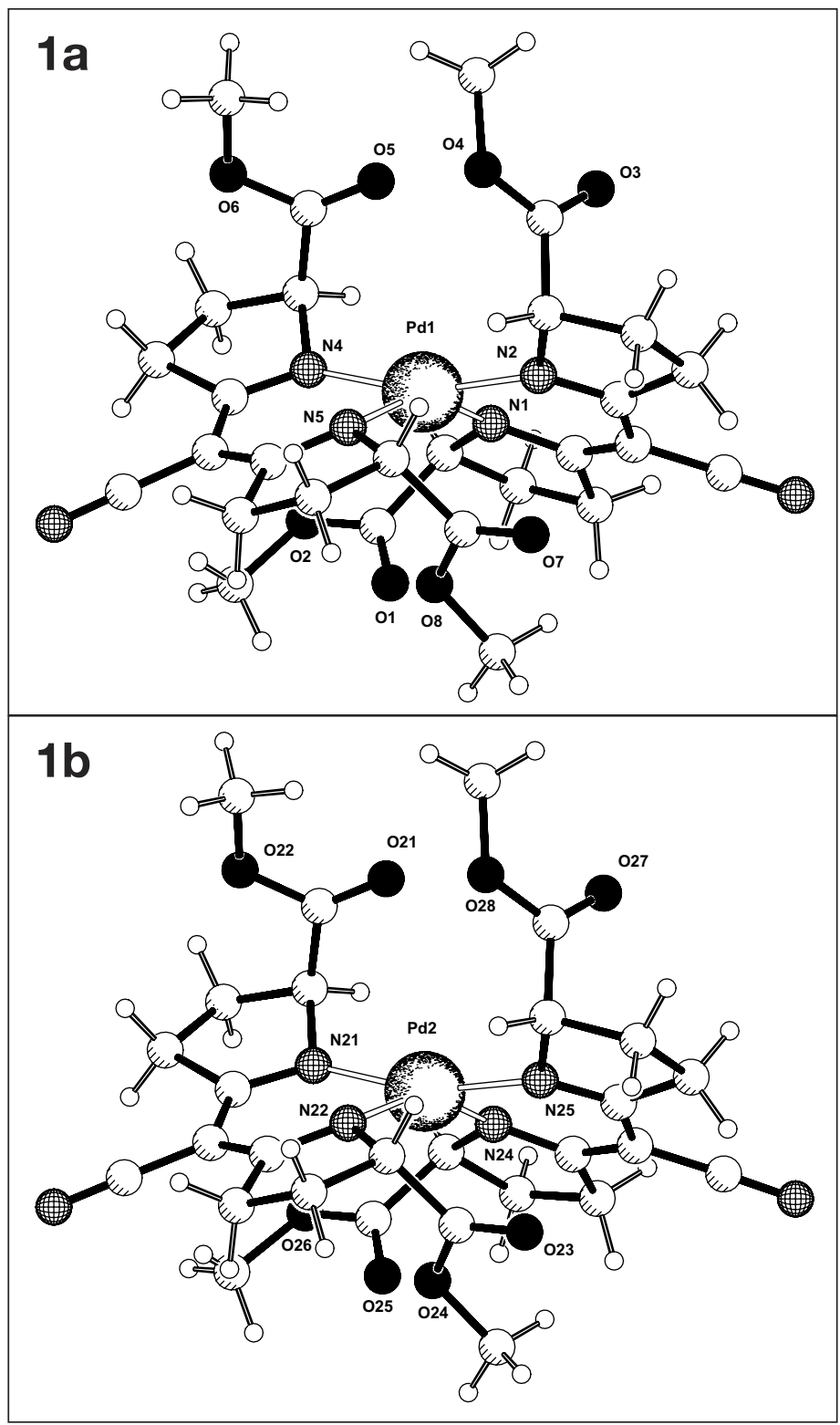

Fig. 1. a). Molecular structure of molecule $\mathrm{A}$ of $\left[\mathrm{Pd}((\mathrm{S}, S)-1)_{2}\right]$. Bond distances $(\AA) \operatorname{Pd}(1)-\mathrm{N}(1)$ 2.045(5), $\operatorname{Pd}(1)-\mathrm{N}(2)$ 2.011(6), $\mathrm{Pd}(1)-\mathrm{N}(4)$ 1.922(5), $\mathrm{Pd}(1)-\mathrm{N}(5)$ 2.056(4); bond angles ( $\left.{ }^{\circ}\right) \mathrm{N}(2)-\mathrm{Pd}(1)-\mathrm{N}(1), 89.5(2), \mathrm{N}(4)-\mathrm{Pd}(1)-$ $\mathrm{N}(1)$ 93.9(2), N(4)-Pd(1)-N(5) 88.15(19), N(2)-Pd(1)-N(5) 96.7(2), N(2)-Pd(1)$\mathrm{N}(4)$ 163.07(19), $\mathrm{N}(1)-\mathrm{Pd}(1)-\mathrm{N}(5)$ 151.48(18). b) Molecular structure of molecule B of $\left[\operatorname{Pd}((S, S)-1)_{2}\right]$. Bond distances $(\AA) \operatorname{Pd}(2)-\mathrm{N}(21) 2.010(5)$, $\mathrm{Pd} / 2)-\mathrm{N}(22)$ 2.054(5), $\mathrm{Pd}(2)-\mathrm{N}(24)$ 2.045(5), $\mathrm{Pd}(2)-\mathrm{N}(25)$ 2.014(6); bond angles ( $\left.{ }^{\circ}\right) \mathrm{N}(21)-\mathrm{Pd}(2)-\mathrm{N}(22)$ 88.8(2), N(21)-Pd(2)-N(24) 92.9(2), N(24)-Pd(2)$\mathrm{N}(25) \quad 89.1(2), \quad \mathrm{N}(22)-\mathrm{Pd}(2)-\mathrm{N}(25) \quad 96.9(2), \quad \mathrm{N}(21)-\mathrm{Pd}(2)-\mathrm{N}(25) \quad$ 163.8(19), $\mathrm{N}(22)-\operatorname{Pd}(2)-\mathrm{N}(24) 152.12(18)$. ative to one another by $62^{\circ}$. This structure is very similar to that obtained for the two independent molecular forms (molecule A and molecule B) found in the crystal of the homochiral $\left[\operatorname{Pd}((S, S)-\mathbf{1})_{2}\right]$. The two molecules are represented in Fig. 1.

As observed for the analogous copper(II) complex [6], the two ligand planes are inclined to each other by an angle of $64.06(12)^{\circ}$ for molecule A and 61.82(12) ${ }^{\circ}$ for molecule B.

The case of the nickel complexes is more complicated. The crystals obtained from an ethanolic solution containing two

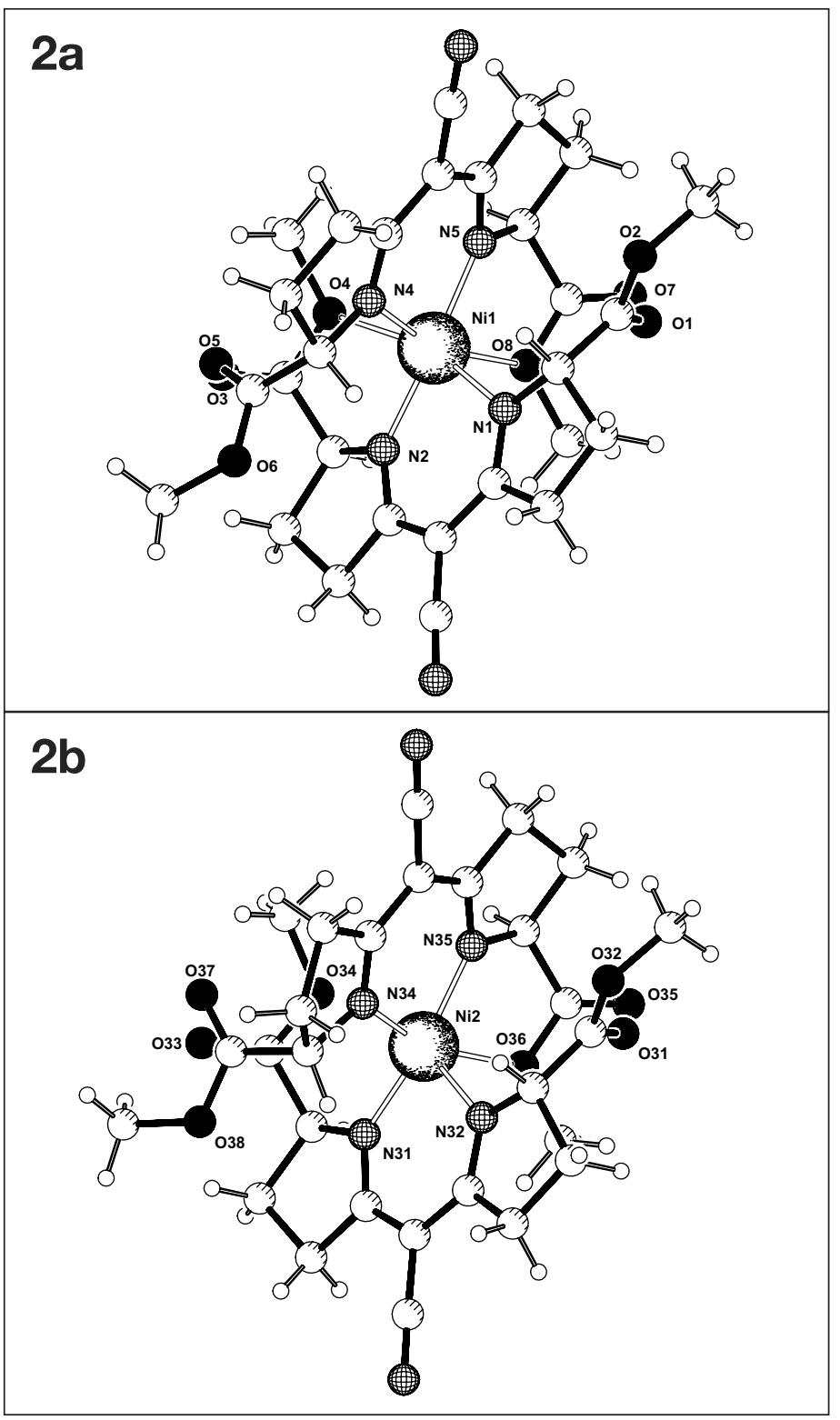

Fig. 2. a) Molecular structure of molecule $A$ of $\Lambda-\left[\mathrm{Ni}((S, S)-1)_{2}\right]$. Bond distances $(\AA ̊) \mathrm{Ni}(1)-\mathrm{N}(1)$ 2.108(3), Ni(1)-N(2) 1.964(4), Ni(1)-N(4) 2.102(4), Ni(1)$\mathrm{N}(5)$ 1.978(4), $\mathrm{Ni}(1)-\mathrm{O}(4) 2.413(3), \mathrm{Ni}(1)-\mathrm{O}(13) 2.448(4)$; bond angles $\left(^{\circ}\right) \mathrm{N}(1)-$ $\mathrm{Ni}(1)-\mathrm{N}(2) \quad 89.10(17), \mathrm{N}(1)-\mathrm{Ni}(1)-\mathrm{N}(5)$ 108.73(16), N(4)-Ni(1)-N(5) 89.9(2), $\mathrm{N}(2)-\mathrm{Ni}(1)-\mathrm{N}(4) \quad$ 109.37(19), $\quad \mathrm{N}(1)-\mathrm{Ni}(1)-\mathrm{N}(4) \quad 92.56(16), \quad \mathrm{N}(2)-\mathrm{Ni}(1)-\mathrm{N}(5)$ 153.42(18). b) Molecular structure of molecule $B$ of $\Lambda$-[Ni $\left.((S, S)-1)_{2}\right]$. Bond distances (Å) Ni(2)-N(31) 1.957(4), Ni(2)-N(32) 1.988(3), Ni(2)-N(34) 1.992(4), $\mathrm{Ni}(2)-\mathrm{N}(35)$ 1.987(4), $\mathrm{Ni}(2)-\mathrm{O}(34)$ 2.392(3), Ni(2)-O(36) 2.640(4); bond angles ( $\left.{ }^{\circ}\right) \mathrm{N}(31)-\mathrm{Ni}(2)-\mathrm{N}(32) 89.59(17), \mathrm{N}(31)-\mathrm{Ni}(2)-\mathrm{N}(35)$ 148.91(19), N(34)-Ni(2)$\mathrm{N}(55)$ 90.86(19), N(32)-Ni(2)-N(34) 92.75(17), N(31)-Ni(2)-N(34) 114.52(18), $\mathrm{N}(32)-\mathrm{Ni}(2)-\mathrm{N}(35)$ 107.65(16). 
equivalents of the optically active ligand crystallise with two independent molecules per asymmetric unit. Molecule A is hexacoordinated, each ligand molecule is, in addition to the two nitrogen atoms, coordinated by one ester group. The coordination occurs via the oxygen atom of the methoxy group. Molecule B, on the other hand shows pentacoordination with only one ligand molecule coordinated by one of its ester groups, again via the methoxy oxygen. The influence on the length of the Ni-N bonds by this additional coordination is rather small. In both molecules the Ni-N bond of the fivemembered ring carrying the coordinated ester group is slightly shortened by about $2 \%$ with respect to the nitrogen atoms of the five-membered rings with the non-coordinated ester group. On the other hand, strong distortion from a regular tetrahedral arrangement of the four nitrogen atoms occurs due to the coordination of one or two ester groups. The structures of the pentaand hexacoordinated forms are shown in Fig. 2.

The case of the $\mathrm{Ni}^{2+}$ complex, obtained from an alcoholic solution of the racemic ligand, shows some interesting features. From selectivity data one would expect the racemic $[\mathrm{Ni}((R, R)-\mathrm{L}(S, S)-\mathrm{L})]$ to crystallise from solution, the equilibrium concentration of which being about three times as high as that of the homochiral compounds (cf. Table). This is obviously not the case, the solid obtained contains the equimolar mixture of the two homochiral complexes. Despite the identical chemical composition of the respective molecular units of the optically active and the racemic complex, some striking differences are observed in their molecular structures. The racemic complex, which crystallises with one molecule of the solvent, contains only one hexacoordinated molecular form of the complex. Interestingly, the ester group is not coordinated with the oxygen of the methoxy group as in the optically active compound, but by the carbonyl oxygen. The Ni-N bonds of the five-membered rings carrying the ester groups are clearly shortened by about $4 \%$ compared to the other $\mathrm{Ni}-\mathrm{N}$ bonds and the distortion of the $\mathrm{NiN}_{4}$ arrangement is increased. Fig. 3 shows the structure of the enantiomer with $\Delta-(R, R)$ configuration.

As observed with $\mathrm{Ni}^{2+}$, no solid heterochiral complex could be obtained from the racemic ligand with $\mathrm{Zn}^{2+}$, despite that it is present by nearly $80 \%$ in solution. No difference between the ligand molecules is observed by NMR in solution indicating identical coordination of both ligand molecules. On the other hand, for the solid complexes the question arises whether they are

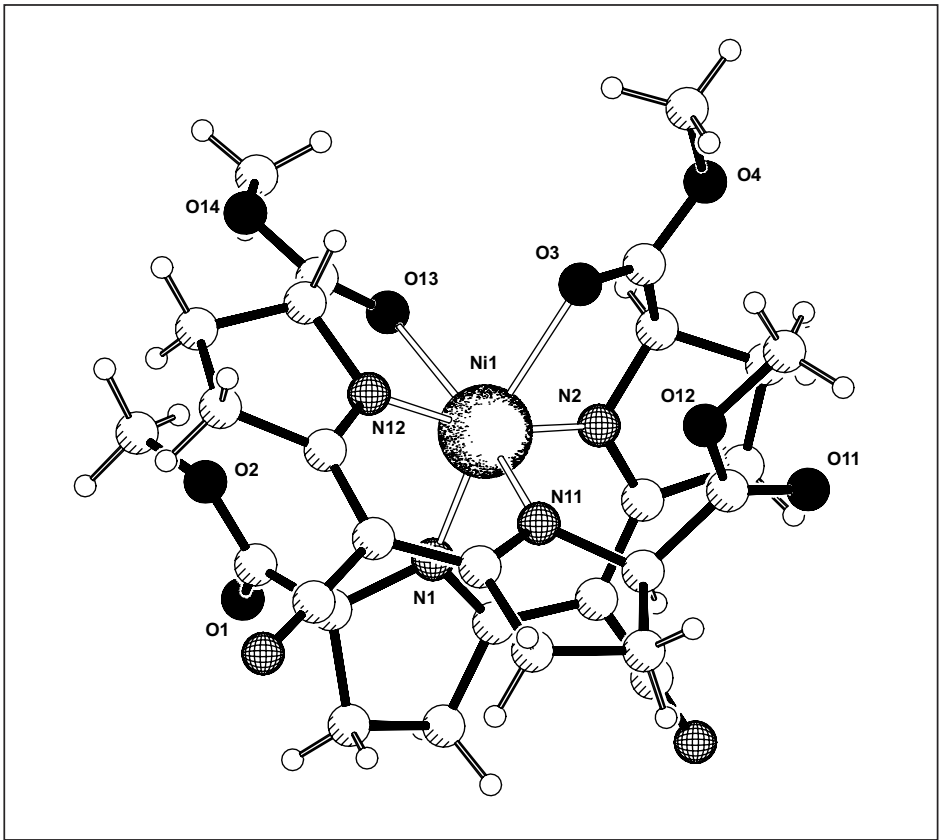

Fig. 3. Molecular structure of the $\Delta-(R, R)$ enantiomer of complex $\{\Delta-[\mathrm{Ni}((R, R)-$ 1) $\left.\left.{ }_{2}\right] \Lambda-\left[\mathrm{Ni}((S, S)-1)_{2}\right]\right\}$. Bond distances (Å) Ni(1)-N(1) 2.053(2), Ni(1)-N(2) 1.972(2), $\mathrm{Ni}(1)-\mathrm{N}(11)$ 2.054(2), Ni(1)-N(12) 1.974(1), Ni(1)-O(3) 2.336(1), Ni(1)-O(13) 2.339(1); bond angles ( $\left.{ }^{\circ}\right) \mathrm{N}(1)-\mathrm{Ni}(1)-\mathrm{N}(2)$ 86.56(7), $\mathrm{N}(11)-\mathrm{Ni}(1)-\mathrm{N}(12)$ 89.88(2), $\mathrm{N}(1)-\mathrm{Ni}(1)-\mathrm{N}(11) \quad 91.09(6), \quad \mathrm{N}(1)-\mathrm{Ni}(1)-\mathrm{N}(12) \quad 104.75(6), \quad \mathrm{N}(2)-\mathrm{Ni}(1)-\mathrm{N}(11)$ 105.47(6) N(2)-Ni(1)-N(12) 163.28(7).

to be considered as tetra- or hexacoordinated. The $\mathrm{Zn}-\mathrm{N}$ bond lengths are almost identical and the distance between the oxygen atoms of the ester group and the coordination center is much longer than in the corresponding $\mathrm{Ni}^{2+}$ complexes. Nevertheless, the non-equivalence of the two substituents of the $\mathrm{C}_{2}$ symmetric ligands and the resulting deviation of the N-Zn-N angles from a regular tetrahedral arrangement indicates some bonding interaction between one ester group of each ligand with the metal ion. The molecular structure of the optically active complex is shown in Fig. 4 and that of the racemic compound in Fig. 5. The presence of two methoxy oxygens near the $\mathrm{Zn}^{2+}$ centre increases the tetrahedral N(1)-Zn$\mathrm{N}(12)$ angle to $128.4^{\circ}$, whereas a similar distortion to $132.8^{\circ}$ is observed for the $\mathrm{N}(1)-\mathrm{Zn}-(\mathrm{N} 1)^{\prime}$ angle in the racemic compound due to the presence of the carbonyl oxygen atoms. This distortion allows the attribution of $\Lambda-(S, S)$ chirality to the optically active and $\Delta-(S, S) / \Lambda-(R, R)$, chirality to the racemic complex, opposite to what is observed for the corresponding $\mathrm{Ni}^{2+}$ complexes with the same coordination pattern.

\section{Discussion}

Stereoselectivity data for the equilibrium reactions in methanolic solutions indicated a clear change in the basic structure of the 1:2 complexes of 1,9-disubstituted semicorrines with the metal ions $\mathrm{Cu}^{2+}$ and
$\mathrm{Pd}^{2+}$ on one side and $\mathrm{Co}^{2+}, \mathrm{Ni}^{2+}$, and $\mathrm{Zn}^{2+}$ on the other. According to Scheme 2, square planar structures are expected for the former and tetrahedral structures for the latter, in agreement with the usual coordination pattern for tetracoordination of these metal ions. Even if one needs to be careful in comparing solid state structures with the behaviour of labile systems in solution, some interesting features arise from the present measurements. Unfortunately, no heterochiral complex, which clearly exists in solution, could be obtained in the solid state for any of the metals studied. This is easily explained for $\mathrm{Pd}^{2+}$ the equilibrium in solution being strongly in favour of the homochiral form. On the other hand, with $\mathrm{Ni}^{2+}$ and $\mathrm{Zn}^{2+}$, despite the higher stability of the heterochiral form, only the homochiral complex crystallises from the solution containing the racemic ligand.

The structural influence of the substituents is clearly visible in the complexes with metal ions favouring tetrahedral and square planar arrangements as well, but seems especially high for the latter leading to angles of $64^{\circ}$ resp. $62^{\circ}$ between the ligand planes in the $\mathrm{Pd}^{2+}$ compound. In the $\mathrm{Zn}^{2+}$ complex, for which a tetrahedral arrangement is expected, the observed angle formed by the two ligand planes is $82^{\circ}$, corresponding to a deviation of only $8^{\circ}$ from a regular arrangement, as it should exist in the corresponding heterochiral compound. The different stereochemical influence of the substituents for metals 


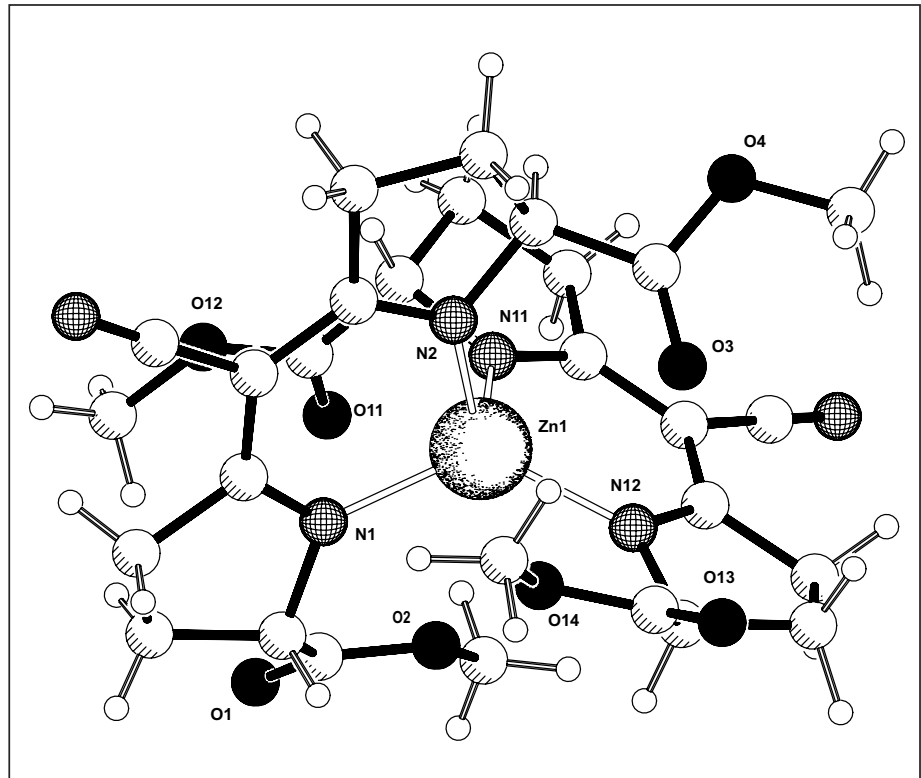

Fig. 4. Molecular structure of $\Lambda$ - $\left[\mathrm{Zn}((S, S)-1)_{2}\right]$. Bond distances $(\AA ̊) \mathrm{Zn}(1)-\mathrm{N}(1)$ 2.001(3), Zn(1)-N(2) 2.002(3), Zn(1)-N(11) 2.027(3), Zn(1)-N(12) 1.989(2), $\mathrm{Zn}(1)-\mathrm{O}(2)$ 2.895(2), $\mathrm{Zn}(1)-\mathrm{O}(14) 2.733$ (2); bond angles $\left(^{\circ}\right) \mathrm{N}(1)-\mathrm{Zn}(1)-\mathrm{N}(2)$ 93.80(11), N(11)-Zn(1)-N(12) 92,66(11), N(1)-Zn(1)-N(11) 114.14(11), N(1)$\mathrm{Zn}(1)-\mathrm{N}(12)$ 128.37(9), N(2)-Zn(1)-N(11) 100.86(11), N(2)-Zn(1)-N(12) 124.83 (11).

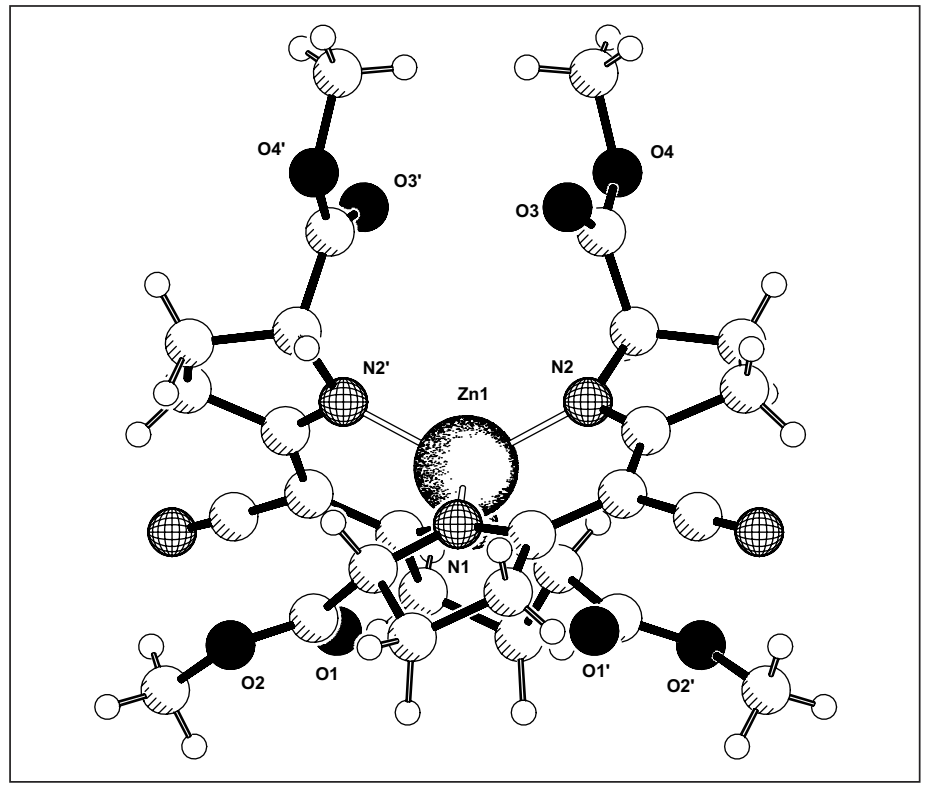

Fig. 5. Molecular structure of the $\Lambda-(R, R)$ enantiomer of complex $\{\Delta$ $\left.\left[\mathrm{Zn}((S, S)-1)_{2}\right] \Lambda-\left[\mathrm{Zn}((R, R)-1)_{2}\right]\right\}$, which possesses $\mathrm{C} 2$ symmetry. Bond distances $(\AA ̊) Z n(1)-(N 1)$ 1.997(3), Zn(1)-(N2) 1.982(2); bond angles $\left(^{\circ}\right) \mathrm{N}(1)-$ $\mathrm{Zn}(1)-\mathrm{N}(2)$ 93.28(10), N(1)-Zn(1)-N(1') 132.84(14), N(1)-Zn(1)-N(2') 108.26(10), $\mathrm{N}(2)-\mathrm{Zn}(1)-\mathrm{N}\left(2^{\prime}\right)$ 124.82(14). favouring square planar arrangements as $\mathrm{Cu}^{2+}$ and $\mathrm{Pd}^{2+}$ compared to metal ions of tetrahedral coordination geometries like $\mathrm{Co}^{2+}, \mathrm{Ni}^{2+}$, and $\mathrm{Zn}^{2+}$, is in agreement with the observed strong negative stereoselectivity of the former, and only moderate preference of the heterochiral compounds of the latter. Similar results have recently been reported for $\mathrm{Cu}^{2+}$ and $\mathrm{Ni}^{2+}$ with the chiral ligand 1,2-bis(2-benzimidazolyl)-1,2-ethanediol [9].

Finally, the results reported in this work show that the realisation of an asymmetric amplification according to Scheme 1, using semicorrin metal complexes as a catalytic system, strongly depends on the metal ion involved. With metal ions favouring tetrahedral ligand arrangements, like $\mathrm{Co}^{2+}$, $\mathrm{Ni}^{2+}$, and $\mathrm{Zn}^{2+}$ among others, asymmetric amplification would be possible. On the other hand, with metal ions preferring the square planar coordination geometry, a negative effect would be observed.

\section{Supplementary Data}

Crystallographic data (excluding structure factors) for the five structures described here are deposited with the Cambridge Crystallographic Data Centre as supplementary publication nos. CCDC 212279 (PdSS1), CCDC 212280 (NiSS1), CCDC 212281 (NiRRSS1), CCDC 212282 (ZnSS1), CCDC 212283 (ZnSSRR1). Copies of the data can be obtained free of charge on application to the Cambridge
Crystallographic Data Centre, 12 Union Road, Cambridge CB2 1EZ, UK [Fax: (internat.) +44-1223/336-033; E-Mail: deposit@ccds.cam.ac.uk].

Received: July 14, 2003

[1] C. Puchot, O. Samuel, E. Dunach, S.H. Zhao, C. Agami, H.B. Kagan, J. Am. Chem. Soc. 1986, 108, 2353.

[2] D. Guillsaneux, S.H. Zhao, D. Rainford, H.B. Kagan, J. Am. Chem. Soc. 1994, 116, 9430; C. Girard, H.B. Kagan, Angew. Chem., Int Ed. 1998, 37, 2922.

[3] N. Kitamura, S. Okada, S. Suga, R. Noyori, J. Am. Chem. Soc. 1989, 111, 4028; R. Noyori, M. Kitamura, Angew. Chem. Int. Ed. Engl. 1991, 30, 49; N. Iwasawa, Y. Hayashi, H. Sakurai, K. Narasaka, Chem. Lett. 1989, 1581; M. Terada, K. Mikami, T. Nakai, J. Chem. Soc., Chem. Commun. 1990, 1623; K. Mikami, M. Terada, S. Narisada, T. Nakai, Synlett 1992, 255; M. Mikami, J. Chem. Soc., Chem. Commun. 1994, 833; K. Mikami, Y Motoyama, M. Terada, Inorg. Chim. Acta 1994, 222, 71; C. Bolm, Tetrahedron: Asymmetry 1991, 2, 701; C. Bolm, G. Schlinloff, K. Harms, Chem. Ber. 1992, 125, 1191; C. Bolm, M. Ewald, M. Felder, Chem. Ber. 1992, 125, 1205; C. Bolm, J. Müller, G. Schlingloff, M. Zehnder, M. Neuburger, J. Chem. Soc., Chem. Commun. 1993, 182; S. Kanemasa, Y. Oderaptoshi, S. Sakaguchi, H. Yamamoto, J. Tanaka, E. Wada, D.P. Curran, J. Am. Chem Soc. 1998, 120, 3074; K. Tanaka, J. Matsui, Y. Kawabata, H. Suzuki, A. Wanatabe, J. Chem. Soc., Chem. Commun. 1991, 1632; B.E. Rossiter, G. Miao, N.M. Swingle, N. Eguchi, A.E. Hernandez, R.G. Patterson, Tetrahedron: Asymmetry, 1992,
2, 231; H. Sasai, T. Suzuki, M. Itoh, M. Shibasaki, Tetrahedron Lett. 1993, 34, 851; N. Kotmasu, M. Hashizume, T. Sugita, S. Uemura, J. Org. Chem. 1993, 58, 4529; G.E. Heck, D. Krishnamurphy, M.C. Grier, J. Org. Chem. 1993, 58, 6543; D.A. Evans, S.G. Nelson, M.R. Cagné, A.R. Muci, J. Am. Chem. Soc. 1993, 115 , 9800; Q.L. Zhou, A. Pfaltz, Tetrahedron 1994, 50, 4467; R.W. Barnhardt, X. Wang, P. Noheda, S.H. Bergens, J. Whelan, B. Bosnich, Tetrahedron 1994, 50, 4335; A.H.M. de Vries, J.F.G.A. Jansen, B.L Feringa, Tetrahedron 1994, 50, 4479; M. Kitamura, S. Suga, R. Noyori, J. Am. Chem. Soc. 1998, 120, 9800; H.B. Kagan, Synlett 2001, 880; H.B. Kagan, Adv. Synth. Cat. 2001, 243, 227; T. Ohga, S. Umeda, Y. Kwanami, Tetrahedron 2001, 57, 4825.

[4] D.G. Blackmond, C.R. McMillan, S. Ramdeehul, A. Schorm, J.M. Brown, J. Am. Chem Soc. 2001, 123, 10103; D.G. Blackmond, Acc. Chem. Res. 2000, 33, 402; D.G. Blackmond, T. Rosner, T. Neugebauer, M.T. Raetz, Angew. Chem., Int. Ed. 1999, 38, 2196; D.G. Blackmond, J. Am. Chem. Soc. 1997, 119, 12934 and 1998, 120, 13349.

[5] C.W. Moeder. M.A. Withener, J.R. Sowa, Jr., J. Am. Chem. Soc. 2000, 122, 7218.

[6] H. Fritschi, U. Leutenegger, K. Siegmann, A. Pfaltz, W. Keller, C. Kratky, Helv. Chim. Acta 1988, 71, 1541.

[7] K. Bernauer, S. Bourqui, D. Hugi-Cleary, R. Warmuth, Helv. Chim. Acta 1992, 75, 1288 .

[8] K. Bernauer, F. Gretillat, H. StoeckliEvans, R. Warmuth, Helv. Chim. Acta 1993, 76, 545.

[9] K. Isele, V. Broughton, C.J. Matthews, A. Williams, G. Bernardelli, P. Franz, S. Decurtins, J. Chem. Soc., Dalton Trans. 2002, 3899 . 